\title{
Upregulated hsa_circ_0000129 expression promotes proliferation and migration of breast cancer cells
}

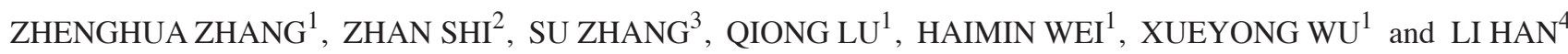 \\ ${ }^{1}$ Department of Oncology, Jing' an District Centre Hospital of Shanghai, Huashan Hospital, \\ Fudan University, Jing'an Branch; ${ }^{2}$ Department of Clinical Oncology, Huadong Hospital, \\ Fudan University; ${ }^{3}$ Department of Traditional Chinese Medicine Jing'an District Centre Hospital of Shanghai, \\ Huashan Hospital, Fudan University, Jing'an Branch; ${ }^{4}$ Department of Traditional Chinese Medicine, \\ Huadong Hospital, Fudan University, Shanghai 200040, P.R. China
}

Received July 13, 2019; Accepted October 20, 2020

DOI: $10.3892 / 01.2021 .12500$

\begin{abstract}
Circular RNAs (circRNAs) are considered potential biomarkers in the pathogenesis and detection of several types of cancer. The present study aimed to investigate the role of hsa_circ_0000129 in the pathogenesis and molecular mechanism underlying breast cancer. A total of 68 pairs of breast cancer and corresponding paracancerous tissue samples, three different breast cancer cell lines (MCF-7, MDA-MB-231 and MDA-MB-468) and a normal human breast cell line (MCF-10A) were used to investigate the expression of hsa_circ_0000129. The effect of hsa_circ_0000129 on cell proliferation, migration and colony formation was assessed in MCF-7 and MDA-MB-468 cells, along with the expression of enhancer of zeste homolog 2 (EZH2). The results demonstrated that hsa_circ_0000129 expression was significantly higher in breast cancer tissues compared with normal tissues. In addition, high hsa_circ_0000129 expression was significantly associated with lymph node metastasis and a higher tumor-node-metastasis stage. Comparisons between the breast cancer cell lines (MCF-7, MDA-MB-231 and MDA-MB-468) and MCF-10A cells indicated similar results. MCF-7 cells overexpressed with hsa_circ_0000129 significantly increased cell proliferation, migration and colony formation compared with
\end{abstract}

Correspondence to: $\mathrm{Dr}$ Xueyong Wu, Department of Oncology, Jing'an District Centre Hospital of Shanghai, Huashan Hospital, Fudan University, Jing'an Branch, 259 Xikang Road, Shanghai 200040, P.R. China

E-mail: wuxy_huashan@126.com

Dr Li Han, Department of Traditional Chinese Medicine, Huadong Hospital, Fudan University, 221 West Yan'an Road, Shanghai 200040, P.R. China

E-mail: hanli_huadong@126.com

Abbreviations: EZH2, enhancer of zeste homolog 2; TNM, tumornode-metastasis; RT, room temperature

Key words: hsa_circ_0000129, breast cancer, EZH2, circular RNAs the negative control group, the effects of which were reversed following hsa_circ_0000129 knockdown in MDA-MB-468 cells. Furthermore, EZH2 expression was positively associated with hsa_circ_0000129 expression. Taken together, the results of the present study suggest that hsa_circ_0000129 may represent a promising prognostic biomarker for breast cancer. In addition, the role of hsa_circ_0000129 in breast cancer cell lines indicates a mechanism for tumorigenesis, as well as a potent target for the treatment of malignant progression.

\section{Introduction}

Breast cancer is one of the most common malignancies affecting women worldwide, whereby $\sim 1$ in every 8 women are diagnosed with breast cancer in their lifetime $(1,2)$. Currently, treatment options for breast cancer include surgery, chemotherapy, radiotherapy and hormone treatment (3). Advancements in the early detection and effective management of breast cancer have resulted in a worldwide decrease in mortalities (3), particularly in western countries, including North America and Europe (1-4). However, breast cancer-associated mortalities continue to increase in developing countries and in patients suffering from different treatment-resistant subtypes of breast cancer (5). In addition, early diagnosis is one of the important factors influencing the long-term survival rate in most types of cancer (3); however, the pathogenesis of breast cancer is complex and heterogenous (6). Computed tomography (CT), magnetic resonance imaging (MRI) and histopathological tools are commonly used for the clinical diagnosis of breast cancer; however, early diagnosis remains relatively uncommon (7). Thus, identifying novel biomarkers and targets for the diagnosis and treatment of breast cancer remains urgent.

Circular RNAs (circRNAs) are a group of non-coding RNAs, which were first discovered in 1976 in RNA viruses via electron microscopy (8). Their unique closed circular structures (lacking 5'-3' untranslated regions and a poly A tail) meant they were initially regarded as functionless by-products of errors in splicing (9). However, several studies have demonstrated that circRNAs influence a number of biological functions, such as microRNA (miRNA) sponging, regulation of transcription, immune regulation and serving as templates 
for protein translation $(8,10,11)$, which may explain their effects on different types of diseases, including cardiovascular diseases, neurological dystrophy and breast, liver, colorectal and lung cancers (12-16).

In breast cancer, extensive dysregulation of several CircRNAs, such as hsa_circ_0104824, CircPVT1 and circRNA 0001073 (17-19), has been reported, and their potential diagnostic and therapeutic values have been demonstrated. For example, 1,705 differentially expressed circRNAs have been identified in breast cancer tissues, and hsa_circ_0001982 has been confirmed to be significantly upregulated in both breast cancer tissues and cell lines (20). In addition, hsa_circ_0001982 knockdown suppresses breast cancer cell proliferation and invasiveness, and induces apoptosis by targeting miR-143, which provides a novel insight into the molecular mechanism underlying breast cancer tumorigenesis (20). Notably, Yin et al (21) identified 41 aberrantly expressed circRNAs in plasma specimens between five breast cancer samples and tissues from paired healthy volunteers; however, only three circRNAs were validated to be significantly dysregulated, and hsa_circ_0001785 exhibited a better diagnostic value compared with the other two candidates (hsa circ_0108942 and hsa_circ_0068033), and carcinoembryonic antigen (21). Furthermore, hsa_circ_0001785 plasma level was significantly associated with tumor-node-metastasis (TNM) staging, grading of the breast tumor and extent of distant metastasis (21). Thus, the potential role of other circRNAs as diagnostic and prognostic biomarkers, as well as therapeutic targets in breast cancer, warrants further research.

In the present study, the novel circRNA hsa_circ_0000129, located at 151145974-151149507 in chromosome 1, was selected using a Gene Expression Omnibus (GEO; https://www.ncbi. nlm.nih.gov/geo/query/acc.cgi?acc=GSE111504) microarray dataset (GSE111504) (22). The present study investigated the expression level of hsa_circ_0000129 in breast cancer patients and breast cancer cell lines, and the results demonstrated that its levels were significantly enhanced in breast cancer tissues and cells compared with their corresponding controls. The effects of hsa_circ_0000129 on the proliferation, migration and colony formation abilities of breast cancer cells and the expression of enhancer of zeste homolog 2 (EZH2) were also studied, aiming to reveal the role of hsa_circ_0000129 in breast cancer progression.

\section{Materials and methods}

Collection of specimens. Breast cancer tissues and adjacent paracancerous tissues (collected $\geq 5 \mathrm{~cm}$ from the tumor border) were collected from 68 patients with breast cancer at the Jing'an District Centre Hospital of Shanghai (Shangai, China) between February 2016 and May 2018. The fresh biopsy tissues were preserved in RNA fixer reagent (Bioteke Corporation) and stored at $-80^{\circ} \mathrm{C}$ prior to subsequent experimentation. Clinical and demographic data were collected from all patients, and no patients had received chemotherapy or radiotherapy prior to surgical resection. Tumor size was determined using CT scan images and both MRI and CT scans were used to diagnose the extent of lymph node metastasis. Tumor staging and grading were assessed according to the cancer staging manual of American Joint Committee on Cancer (7th edition) (23). The present study was approved by the Human Research Ethics Committee of Huashan Hospital (Shanghai, China; approval no. 2016MS02), and written informed consent was provided by all patients prior to the study start.

Cell culture. The breast cancer cell lines (MCF-7, MDA-MB-231 and MDA-MB-468) and normal epithelial breast cell line (MCF10A) were purchased from the American Type Culture Collection. All cells were maintained in DMEM supplemented with $10 \%$ fetal bovine serum (FBS) and $1 \%$ penicillin-streptomycin (all purchased from Gibco; Thermo Fisher Scientific, Inc.), at $37^{\circ} \mathrm{C}$ with $5 \% \mathrm{CO}_{2}$.

Cells successfully transfected with the overexpression vector or interference sequence of hsa_circ_0000129 were cultured in complete DMEM at $37^{\circ} \mathrm{C}$.

Transient transfection. MCF-7 and MDA-MB-468 cells were seeded into 6 -well plates at a density of $4.0 \times 10^{5}$ cells $/$ well. After $24 \mathrm{~h}, \mathrm{MCF}-7$ cells were transfected with the overexpression vector for hsa_circ_0000129 (Geneseed Biotech, Inc.) using Lipofectamine ${ }^{\circledR} 2000$ reagent (Invitrogen; Thermo Fisher Scientific, Inc.), according to the manufacturer's protocol, while a control vector (Geneseed Biotech, Inc.) was used as the negative control (NC). MDA-MB-468 cells were transfected with an interference sequence for hsa_circ_0000129 or NC sequence (Geneseed Biotech, Inc., http://www.geneseed.com.cn), using Lipofectamine ${ }^{\circledR} 2000$ reagent. All cells were transfected for $24 \mathrm{~h}$ at $37^{\circ} \mathrm{C}$ and another $48 \mathrm{~h}$ later, transfection efficiency was determined via reverse transcription-quantitative (RT-q)PCR analysis.

$R T-q P C R$. Total RNA was extracted from breast cancer tissues and adjacent paracancerous tissues using TRIzol ${ }^{\circledR}$ reagent (Invitrogen; Thermo Fisher Scientific, Inc.), according to the manufacturer's protocol. Total RNA was reverse transcribed into double-strand cDNA using the ReverTra Ace qPCR RT kit (cat. no. FSQ-101; Toyobo Life Science), according to the manufacturer's protocol. qPCR was subsequently performed using the SYBR Premix Ex Taq ${ }^{\mathrm{TM}}$ II kit (cat. no. RR820A; Takara Biotechnology Co., Ltd.), $0.5 \mu \mathrm{l}$ cDNA, $0.5 \mu \mathrm{l}$ each primer and $5 \mu \mathrm{l}$ SYBR Green. The following primer sequences were designed by Sangon Biotech Co., Ltd., and used for qPCR: GAPDH forward, 5'-GGAGCGAGATCCCTCCAAAAT-3' and reverse, 5'-GGCTGTTGTCATACTTCTCATGG-3'; hsa_circ_0000129 forward, 5'-AAGAGGGAAATCCCAGCA GA-3' and reverse, 5'-GCATGAGGAGTCAATGCAGA-3'; and EZH2 forward, 5'-AATCAGAGTACATGCGACTGAGA-3' and reverse, 5'-GCTGTATCCTTCGCTGTTTCC-3'. The following thermocycling conditions were used for qPCR: $94^{\circ} \mathrm{C}$ for $5 \mathrm{~min}$, 42 cycles of $94^{\circ} \mathrm{C}$ for $5 \mathrm{sec}$ and $60^{\circ} \mathrm{C}$ for $1 \mathrm{~min}$. Relative expression levels were calculated using the $2^{-\Delta \Delta \mathrm{Cq}}$ method (24) and normalized to the internal reference gene GAPDH.

Cell proliferation assay. MCF-7 and MDA-MB-468 cells transfected with NC or hsa_circ_0000129 sequences were seeded into 96 -well plates at a density of $3.5 \times 10^{3}$ cells/well and incubated at $37^{\circ} \mathrm{C}$ for $1-7$ days. Subsequently, cells were incubated with $5 \mathrm{mg} / \mathrm{ml}$ MTT reagent (Sigma-Aldrich; Merck KGaA) for $3 \mathrm{~h}$ at $37^{\circ} \mathrm{C}$. Following the MTT incubation, the supernatant was removed and the purple formazan crystals were dissolved using 
A

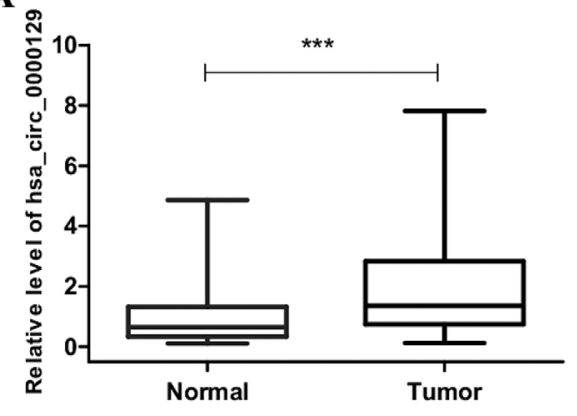

B ㅇำ

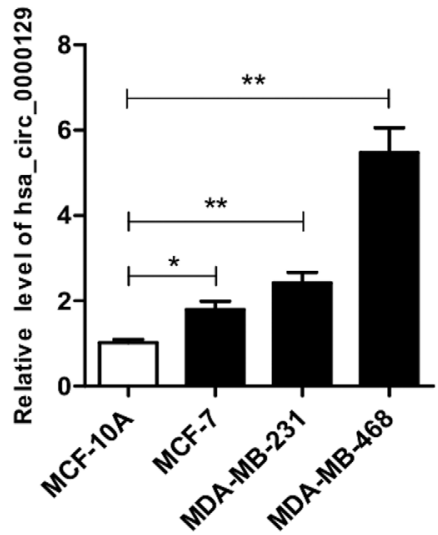

Figure 1.hsa_circ_0000129 expression in breast cancer tissues and cell lines. (A) hsa_circ_0000129 expression in 68 pairs of breast cancer tissues and normal breast tissues. (B) hsa_circ_0000129 expression in breast cancer cell lines (MCF-7, MDA-MB-231 and MDA-MB-468) and normal epithelial breast cell line (MCF-10A). ${ }^{*} \mathrm{P}<0.05,{ }^{* *} \mathrm{P}<0.01,{ }^{* * *} \mathrm{P}<0.001$. Circ, circular.

dimethyl sulfoxide (Sigma-Aldrich; Merck KGaA). Cell proliferation was subsequently analyzed at a wavelength of $492 \mathrm{~nm}$, between 0-7 days. Cell proliferation was assessed using the following formula: Proliferation rate $=\mathrm{A}(\mathrm{sample}) / \mathrm{A}(\mathrm{control})$, where $\mathrm{A}$ is the absorbance measured at $492 \mathrm{~nm}$ and control is the sample which was analyzed on day 0 .

Colony formation assay. MCF-7 and MDA-MB-468 cells transfected with NC or hsa_circ_0000129 sequences were seeded into 6 -well plates at a density of $6.0 \times 10^{2}$ cells/well and incubated for 14 days at $37^{\circ} \mathrm{C}$. The cell media were replaced every $48 \mathrm{~h}$. The plates were subsequently washed three times with PBS and the cell colonies were fixed with $100 \%$ methanol at room temperature for $15 \mathrm{~min}$, prior to staining with $1 \%$ crystal violet for $30 \mathrm{~min}$ at room temperature. Stained colonies (with $>50$ cells) were observed under a light microscope (magnification, x200).

Migration assay. The migratory ability MCF-7 and MDA-MB-468 cells transfected with NC or hsa_circ_0000129 sequences was assessed using 24 -well Transwell inserts $(8-\mu \mathrm{m}$ pore size; EMD Millipore), according to the manufacturer's protocol. A total of $1.0 \times 10^{4}$ cells were plated in the upper chambers of Transwell plates in $100 \mu 1$ serum-free DMEM. DMEM $(600 \mu \mathrm{l})$ supplemented with $10 \%$ FBS was plated in the lower chambers. Following incubation at $37^{\circ} \mathrm{C}$ for $24 \mathrm{~h}$, the non-migratory cells in the upper chambers were removed using cotton swabs, while the migratory cells in the lower chambers were fixed with $100 \%$ methanol at room temperature for $15 \mathrm{~min}$ and stained with $1 \%$ crystal violet for $30 \mathrm{~min}$ at room temperature. Stained cells were counted in five randomly selected fields using a light microscope (magnification, x200).

Western blotting. Cell lysates were extracted from MCF-7 and MDA-MB-468 cells transfected with NC or hsa_circ_0000129 sequences using cell lysis buffer (Sigma-Aldrich; Merck KGaA), and centrifuged at $13,800 \mathrm{x}$ g for $5 \mathrm{~min}$ at $4^{\circ} \mathrm{C}$. Total protein was quantified using the BCA protein assay kit (cat. no. 70-PQ0011; Hangzhou MultiSciences Biotech Co., Ltd., http://www. liankebio.com), according to the manufacturer's protocol, and $20 \mu \mathrm{g}$ protein/lane was separated via SDS-PAGE on a $12 \%$ gel. The separated proteins were subsequently transferred onto polyvinylidene fluoride membranes and blocked with $5 \%$ non-fat milk at room temperature for $2 \mathrm{~h}$. The membranes were incubated with primary monoclonal antibodies against EZH2 (cat. no. 5246; 1:1,000) and $\beta$-actin (cat. no. 3700, $1: 1,000)$ overnight at $4^{\circ} \mathrm{C}$. Following the primary incubation, membranes were incubated with HRP-linked secondary antibody (cat. no. 7076, 1:1,000), at room temperature for $1 \mathrm{~h}$ and subsequently visualized using the Ultra-sensitive ECL chemiluminescence kit (cat. no. P0018AS; Beyotime Institute of Biotechnology). All antibodies were purchased from Cell Signaling Technology, Inc.

Statistical analysis. Statistical analysis was performed using SPSS 16.0 (IBM Corp.) and GraphPad Prism 5.0 (GraphPad Software, Inc.). All experiments were performed in triplicate and data are presented as the mean \pm standard deviation. Paired and unpaired Student's t-tests were used to compare differences between two groups, while ANOVA followed by Tukey's post hoc test were used to compare differences between multiple groups. $\mathrm{P}<0.05$ was considered to indicate a statistically significant difference.

\section{Results}

hsa_circ_0000129 expression is upregulated in breast cancer. RT-qPCR analysis was performed to detect hsa_circ_0000129 expression in breast cancer tissues and adjacent normal tissues. The results demonstrated that hsa_circ_0000129 expression was significantly higher in tumor tissues compared with normal tissues $(\mathrm{P}<0.001$; Fig. 1A). Similarly, hsa_ circ_0000129 expression was compared between the breast cancer cell lines (MCF-7, MDA-MB-231 and MDA-MB-468) and normal MCF10A cells. The results demonstrated that hsa circ_0000129 expression was significantly higher in all three cancer cell lines (MCF-7, MDA-MB-231 and MDA-MB-468) compared with MCF10A cells $(\mathrm{P}<0.05, \mathrm{P}<0.01$ and $\mathrm{P}<0.001$, respectively; Fig. 1B).

Association between hsa_circ_0000129 expression and the clinicopathological characteristics of patients with breast cancer. As presented in Table I, significant associations 
Table I. Association between hsa_circ_0000129 expression and the clinicopathological characteristics of patients with breast cancer $(n=68)$.

\begin{tabular}{lccc} 
Characteristic & Number of cases, $n$ & $\begin{array}{c}\text { hsa_circ_0000129 expression, } \\
\text { mean } \pm \text { standard deviation }\end{array}$ & P-value \\
\hline $\begin{array}{l}\text { Age, years } \\
\leq 50\end{array}$ & 36 & $2.15 \pm 1.47$ & 0.499 \\
$>50$ & 32 & $1.87 \pm 1.93$ & 0.348 \\
Menopause & & & \\
$\quad$ No & 40 & $1.97 \pm 1.45$ & 0.847 \\
Yes & 28 & $2.37 \pm 2.07$ & $0.036^{\mathrm{a}}$ \\
Tumor size, cm & 30 & $1.98 \pm 1.41$ & \\
$\leq 2$ & 38 & $2.06 \pm 1.91$ & $0.002^{\mathrm{b}}$ \\
$>2$ & & & $1.58 \pm 1.36$ \\
Lymph node metastasis & 33 & $2.44 \pm 1.89$ & \\
$\quad$ No & 35 & $1.47 \pm 1.33$ & \\
Yes & & $2.68 \pm 1.87$ & \\
TNM stage & 37 & & \\
I-II & 31 & & \\
III-IV & & & \\
\hline
\end{tabular}

${ }^{\mathrm{a}} \mathrm{P}<0.05,{ }^{\mathrm{b}} \mathrm{P}<0.01$. TNM, tumor-node-metastasis.
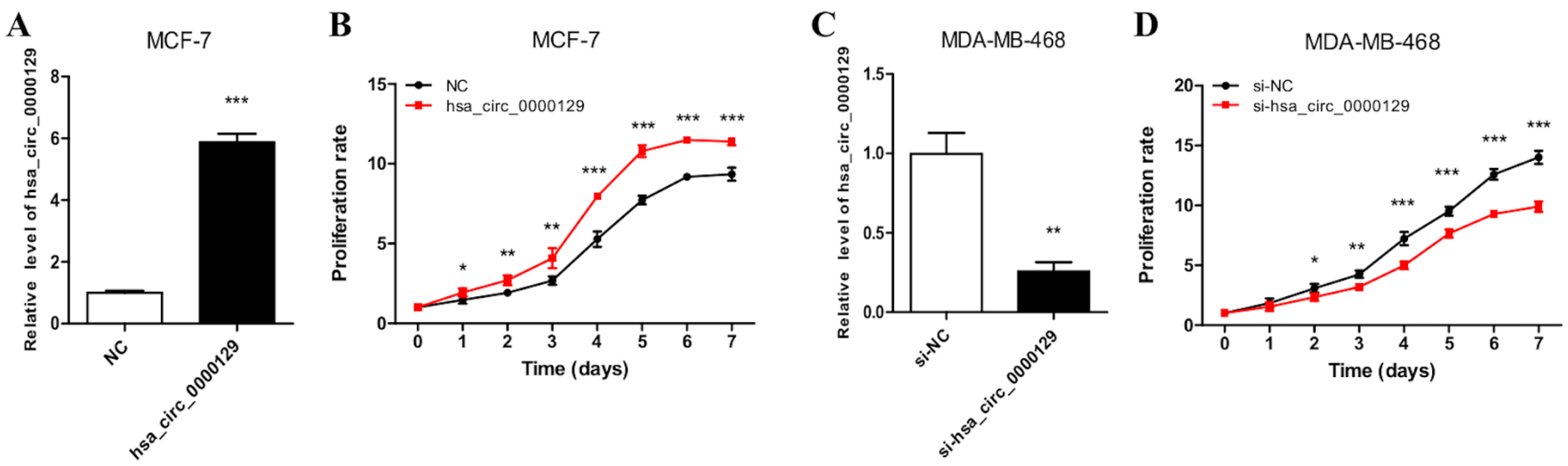

Figure 2. Effect of hsa_circ_0000129 on the proliferation of breast cancer cells. (A) RT-qPCR analysis confirmed hsa_circ_0000129 expression in MCF-7 cells transfected with an overexpression vector. (B) Overexpression of hsa_circ_0000129 promoted MCF-7 cell proliferation. (C) RT-qPCR analysis confirmed hsa_circ_0000129 expression in MDA-MB-468 cells transfected with si-hsa_circ_0000129. (D) hsa_circ_0000129 knockdown inhibited MDA-MB-468 cell proliferation. ${ }^{*} \mathrm{P}<0.05,{ }^{* *} \mathrm{P}<0.01,{ }^{* * *} \mathrm{P}<0.001$ vs. NC group or day 0 group or si-NC group. Circ, circular; RT-qPCR, reverse transcription-quantitative PCR; si, small interfering; NC, negative control.

were observed between hsa_circ_0000129 expression and lymph node metastasis $(\mathrm{P}<0.05)$ and TNM stage $(\mathrm{P}<0.01)$ However, no significant associations were observed between hsa_circ_0000129 expression and age, menopause status and tumor size.

hsa_circ_0000129 regulates the proliferation of breast cancer cells. RT-qPCR analysis demonstrated the successful overexpression of hsa_circ_0000129 in MCF-7 cells (Fig. 2A). MCF-7 cells transfected with hsa_circ_0000129 sequence exhibited significantly increased cell proliferation compared with cells in the $\mathrm{NC}$ group $(\mathrm{P}<0.001$ at days 4, 5, 6 and 7; Fig. 2B). RT-qPCR analysis also demonstrated the successful knockdown of hsa_circ_0000129 in MDA-MB-468 cells (Fig. 2C). The results of the MTT assay demonstrated that hsa_circ_0000129 knockdown significantly decreased the proliferation rate of MDA-MB-468 cells compared with cells in the si-NC group $(\mathrm{P}<0.001$ at days $4,5,6$ and 7 post-incubation; Fig. 2D).

hsa_circ_0000129 regulates the migration of breast cancer cells. The results demonstrated that the migratory ability was significantly enhanced in MCF-7 cells transfected with hsa_circ_0000129 vector compared with the NC group $(\mathrm{P}<0.001$; Fig. 3A). Conversely, the migratory ability was significantly attenuated in MDA-MB-468 cells transfected 
A

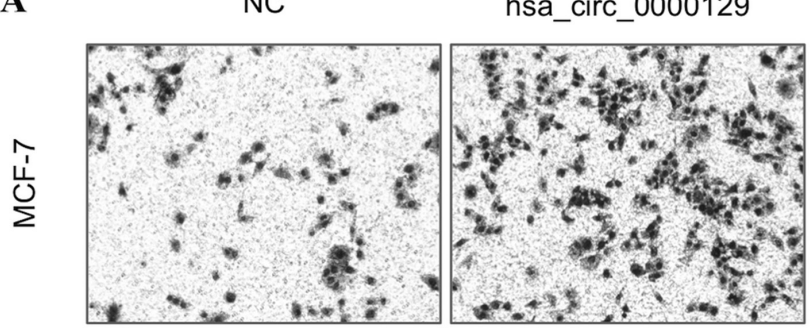

B

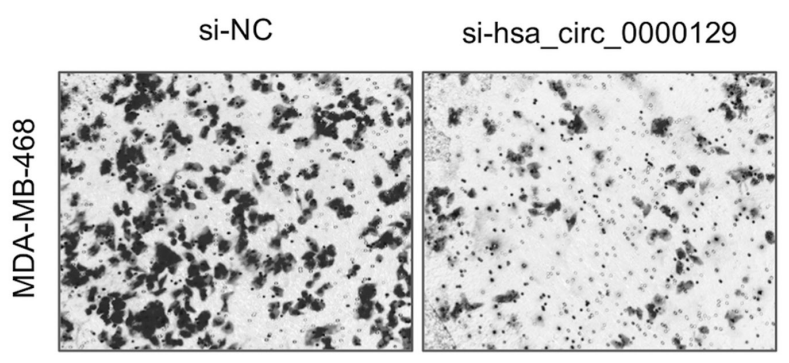

MCF-7

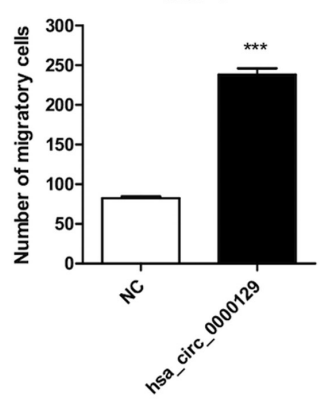

MDA-MB-468

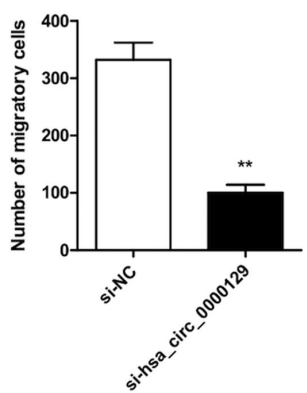

Figure 3. Effect of hsa_circ_0000129 on the migration of breast cancer cells. (A) Overexpression of hsa_circ_0000129 enhanced the migratory ability of MCF-7 cells. (B) hsa_circ_0000129 knockdown inhibited the migratory ability of MDA-MB-468 cells. ${ }^{* *} \mathrm{P}<0.01,{ }^{* * *} \mathrm{P}<0.001$ vs. NC group or si-NC group. Circ, circular; $\mathrm{NC}$, negative control; si, small interfering.

A

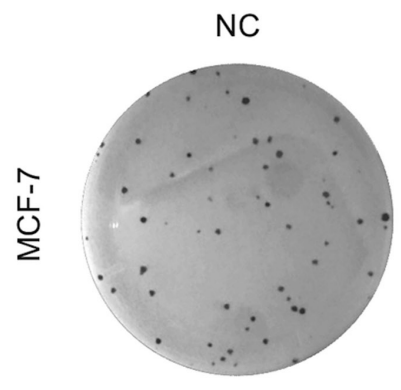

B

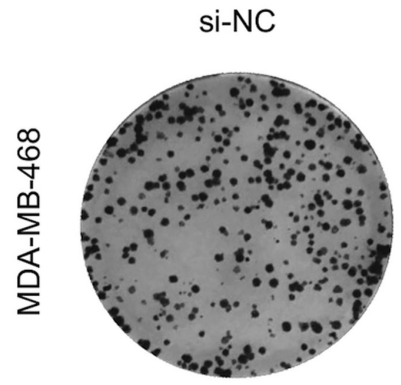

hsa_circ_0000129

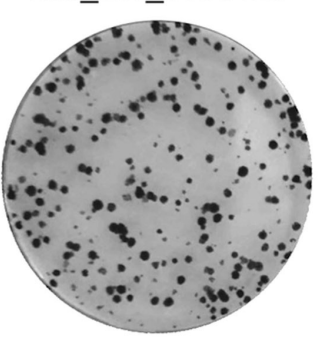

si-hsa_circ_0000129

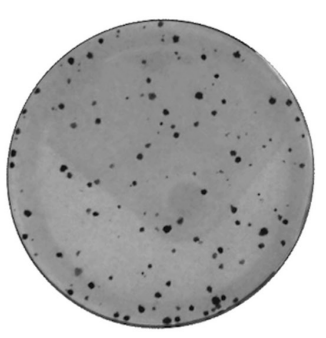

MCF-7

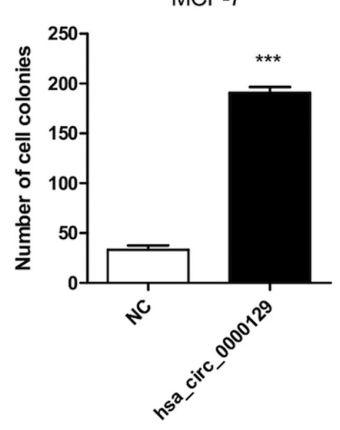

MDA-MB-468

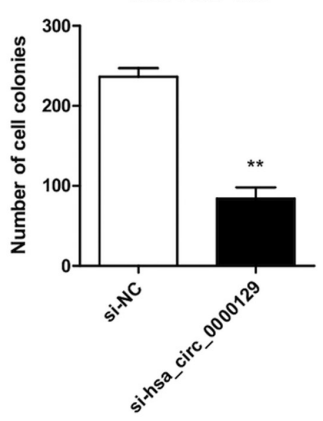

Figure 4. Effect of hsa_circ_0000129 on the colony formation ability of breast cancer cells. (A) Overexpression of hsa_circ_0000129 significantly enhanced the colony formation ability of MCF-7 cells. (B) hsa_circ_0000129 knockdown significantly suppressed the colony formation ability of MDA-MB-468 cells. ${ }^{* *} \mathrm{P}<0.01,{ }^{* * * *} \mathrm{P}<0.001$, vs. NC group or si-NC group. Circ, circular; NC, negative control; si, small interfering.

with si-hsa_circ_0000129 compared with the si-NC group (P<0.01; Fig. 3B).

hsa_circ_0000129 regulates colony formation in breast cancer cells. The effect of hsa_circ_0000129 on colony formation ability was assessed. The results demonstrated that the colony formation ability of MCF-7 cells was significantly enhanced following overexpression of hsa-circ-0000129 $(\mathrm{P}<0.001$; Fig. 4A). Conversely, hsa_circ_0000129 knockdown significantly inhibited the colony formation ability of MDA-MB-468 cells compared with the si-NC group (P<0.01; Fig. 4B). 
A

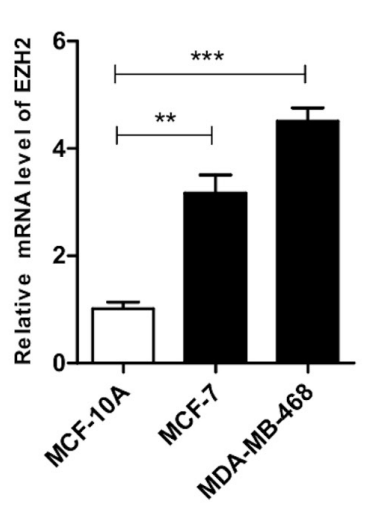

D

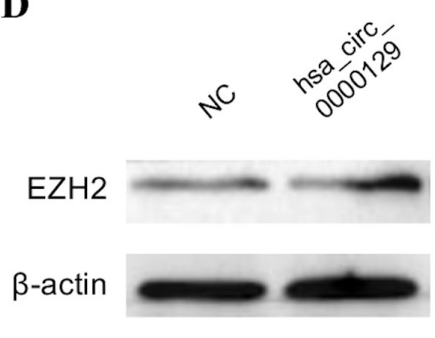

MCF-7
B

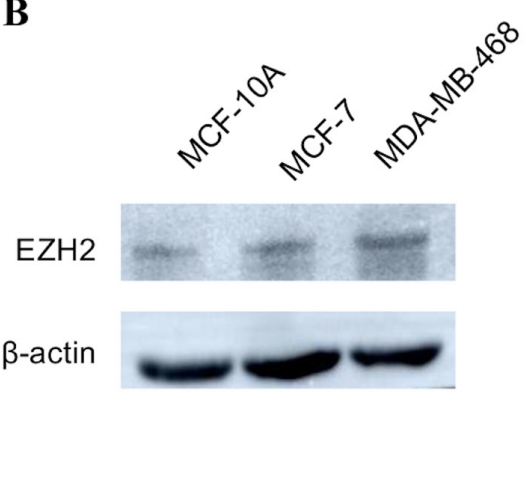

$\mathbf{E}$

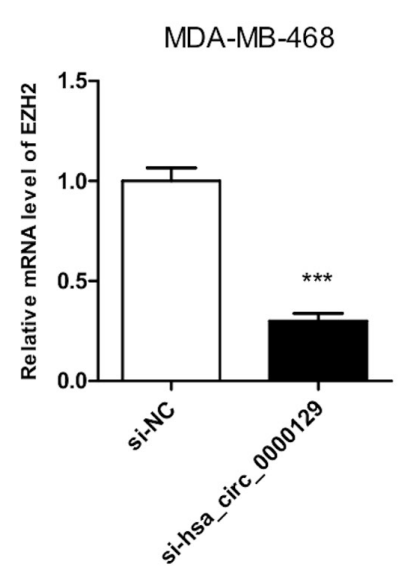

C

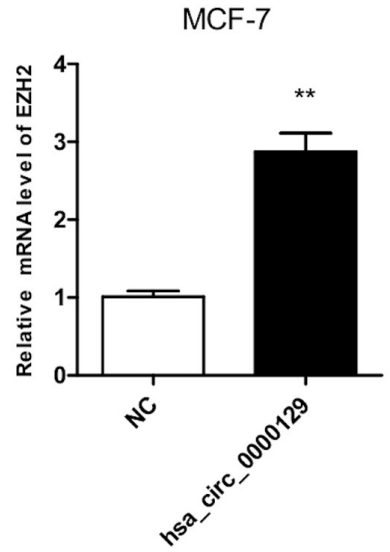

F

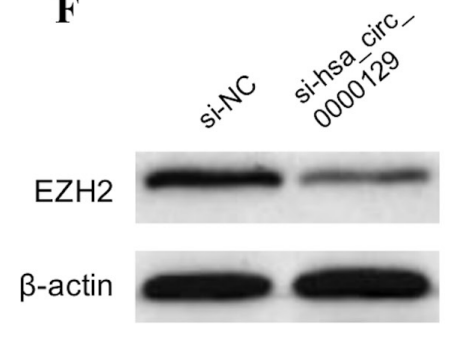

MDA-MB-468

Figure 5. Effect of hsa_circ_0000129 on EZH2 expression. (A) RT-qPCR and (B) western blot analyses demonstrated that EZH2 expression was significantly upregulated in the cancer cell lines compared with normal breast cells. (C) RT-qPCR and (D) western blot analyses demonstrated that overexpression of hsa_circ_0000129 upregulated EZH2 expression in MCF-7 cells. (E) RT-qPCR and (F) western blot analyses demonstrated that hsa_circ_0000129 knockdown downregulated EZH2 expression in MDA-MB-468 cells. ${ }^{* *} \mathrm{P}<0.01,{ }^{* * *} \mathrm{P}<0.001$. Circ, circular; RT-qPCR, reverse transcription-quantitative PCR; NC, negative control; si, small interfering.

Effect of hsa_circ_0000129 on EZH2 expression. EZH2 expression was detected to determine its underlying molecular mechanism in relation to hsa_circ_0000129. RT-qPCR and western blot analyses demonstrated that EZH2 expression was significantly higher in MCF-7 and MDA-MB-468 cells compared with MCF-10A cells $(\mathrm{P}<0.01$ and $\mathrm{P}<0.001$, respectively; Fig. 5A and B). RT-qPCR and western blot analyses also demonstrated that EZH2 expression was significantly higher in MCF-7 cells overexpressing hsa_circ_0000129 compared with cells in the NC group ( $\mathrm{P}<0.01$; Fig. 5C and D). Conversely, RT-qPCR and western blot analyses demonstrated that EZH2 expression was significantly downregulated in MDA-MB-468 cells following hsa_circ_0000129 knockdown compared with cells in the si-NC group according $(\mathrm{P}<0.001$; Fig. 5E and $\mathrm{F})$.

\section{Discussion}

The present study aimed to investigate the role and determine the underlying molecular mechanism of hsa_circ_0000129 in breast cancer. The results demonstrated that hsa_circ_0000129 expression was significantly higher in breast cancer tissues compared with adjacent paracancerous tissues, and hsa circ_0000129 expression was significantly associated with lymph node metastasis and high TNM staging, which suggests that high hsa_circ_0000129 expression may predict a higher malignancy index in patients with breast cancer.

Similarly, the results of the present study demonstrated that hsa_circ_0000129 expression was significantly higher in all three breast cancer cell lines (MCF-7, MDA-MB-231 and MDA-MB-468) compared with the normal epithelial breast cancer cell line, MCF10A. Notably, hsa_circ_0000129 expression was higher in MDA-MB-468 cells compared with MDA-MB-231 cells, and exhibited the lowest expression in MCF-7 cells. The differences in expression between the three breast cancer cell lines may be attributed to the differences in cellular characteristics. First, although all three cell lines originate from the metastatic pleural effusions of patients with breast cancer, the malignancy degrees may vary (25). Secondly, the results of the present study demonstrated that high hsa_circ_0000129 expression in the clinical samples was associated with a higher degree of malignancy, which may also be applicable to these cell lines. Furthermore, certain studies investigating these cell lines have reported that the phenotypes of the cells are notably different, whereby the migratory and invasive abilities, and stemness of MDA-MB-231 cells are higher compared with MCF-7 cells (25-27). In addition, the migratory, invasive and colony formation abilities of MDA-MB-468 cells have been demonstrated to be significantly higher compared with MDA-MB-231 cells (28), which 
are consistent with the results of the present study and suggest that MDA-MB-468 cells are more malignant than MCF-7 cells.

The role of hsa_circ_0000129 in different phenotypes of breast cancer cells was assessed in the present study. The results demonstrated that cell proliferation, migration and colony formation were significantly enhanced in $\mathrm{MCF}-7$ cells overexpressed with hsa_circ_0000129. Conversely, hsa_circ_0000129 knockdown significantly inhibited cell proliferation, migration and colony formation of MDA-MB-468 cells compared with the control cells. Generally, higher proliferative, migratory and colony formation abilities indicate a more malignant phenotype in cancer cells $(29,30)$. Thus, the results of the present suggest that hsa_circ_0000129 expression level in breast cancer plays an important role in cellular malignancy, which is closely associated with the malignancy degree of breast cancer. Taken together, the results of the present study suggest that hsa_circ_0000129 may represent a promising prognostic biomarker for breast cancer.

In accordance with the results of the present study, several studies have investigated the role of circRNAs in breast cancer and other types of cancer, including hepatocellular carcinoma, clear cell renal cell carcinoma and colorectal cancer (8), and demonstrated the potential role of circRNAs as both diagnostic and prognostic markers. For example, Nair et al (31) cataloged the different circRNAs associated with three different types of breast cancer, triple negative, estrogen receptor positive and ErB2 overexpressing Her2-positive breast cancer cells. In addition, Wang et al (32) demonstrated that circ-UBE2D2 is upregulated in breast cancer cell lines and tissues, and is also closely associated with aggressive clinical characteristics and a poor prognosis (32). Furthermore, it has been reported that silencing circ-UBE2D2 notably inhibits the proliferative, migratory and invasive abilities of BC cells, and the in vivo delivery of oligonucleotides that inhibits circ-UBE2D2 significantly delays tumor growth (32). To the best of our knowledge, the present study is the first to investigate the role of hsa_circ_0000129 in breast cancer.

The potential underlying molecular mechanism of hsa_circ_0000129 in breast cancer was assessed in the present study. EZH2, a polycomb group protein, is known to be associated with carcinogenesis $(33,34)$. Polycomb group proteins are evolutionarily conserved regulators and act by silencing different growth regulatory genes $(33,34)$. There are two main families of polycomb repressive complexes, 1 and 2 (PRC2), and EZH2 is a catalytic subunit of PRC2 (34). Previous studies have demonstrated that upregulated EZH2 expression is associated with different types of carcinoma, including ovarian, cervical, glioma, breast, prostate and renal cell cancer (33-35).

Previous studies have reported that upregulated EZH2 expression in breast cancer is associated with advanced form of the disease, higher grade of tumor staging, increased proliferation of tumor, increased chance of metastasis and poor long term overall survival (36-38). Xue et al (39) reported that arsenite, a known carcinogenic agent, upregulates hsa circ_100284 expression in human ketratocyte (HaCaT) cells. The underlying molecular mechanism was also investigated, and it was demonstrated that hsa_circ_100284 serves as a sponge for miR-217 and indirectly upregulates EZH2. Notably, EZH2 upregulates the expression levels of cyclin D1 and K4, and promotes malignant changes in $\mathrm{HaCaT}$ cells. Furthermore, while investigating the association between EZH2 expression and risk of developing breast cancer in a case control study, Beca et al (40) discovered that EZH2 expression is an independent risk factor for breast cancer.

Similarly, the underlying molecular mechanism of hsa_circ_0000129 in breast cancer was investigated in the present study, and the results demonstrated that EZH2 expression was upregulated in all three breast cancer cell lines. In addition, EZH2 expression was significantly enhanced in MCF-7 cells transfected with hsa_circ_0000129 vector, and significantly downregulated in MDA-MB-468 cells following hsa_circ_0000129 knockdown, compared with their respective control groups. Collectively, the results of the present study suggest that hsa_circ_0000129 exerts its role in breast cancer by regulating expression of the oncogene, EZH2.

A limitation of the present study is that $\mathrm{EZH} 2$ expression was only assessed in breast cancer cell lines, while its expression in human breast cancer tissues remains unknown. Thus, this will be the focus of prospective studies.

In conclusion, the results of the present study suggest that hsa_circ_0000129 may represent a prognostic marker for patients with breast cancer. The role of hsa_circ_0000129 in breast cancer cell lines reveals a novel mechanism for tumorigenesis, as well as a potent target for interference of malignant progression. In addition, the carcinogenic molecular mechanism of hsa_circ_0000129 may be associated with upregulated EZH2 expression in breast cancer.

\section{Acknowledgements}

Not applicable.

\section{Funding}

The present study was funded by the Joint Medical Research Program of Shanghai Jing'an District Science and Technology Commission and Health Commission [grant no. (JI)2016MS02] and the National Key R\&D Program of China (grant no. 2018YFC2002400).

\section{Availability of data and materials}

The datasets used and/or analyzed during the present study are available from the corresponding author upon request.

\section{Authors' contributions}

ZZ, ZS and SZ conceived and designed the present study, and analyzed and interpreted the data. XW and LH provided administrative support and interpreted the data. QL and HW performed the experiments, and collected and assembled the data. All authors contributed to drafting the initial manuscript and revising the manuscript for important intellectual content. All authors have read and approved the final manuscript.

\section{Ethics approval and consent to participate}

The present study was approved by the Human Research Ethics Committee of Huashan Hospital (Shanghai, China; approval no. 2016MS02), and written informed consent was provided by all patients prior to the study start. 


\section{Patient consent for publication}

Not applicable.

\section{Competing interests}

The authors declare that they have no competing interests.

\section{References}

1. Wang $X$ and Fang L: Advances in circular RNAs and their roles in breast cancer. J Exp Clin Cancer Res 37: 206, 2018.

2. Key TJ, Verkasalo PK and Banks E: Epidemiology of breast cancer. Lancet Oncol 2: 133-140, 2001.

3. Harbeck N and Gnant M: Breast cancer. Lancet 389: 1134-1150, 2017.

4. Malvezzi M, Carioli G, Bertuccio P, Rosso T, Boffetta P, Levi F, La Vecchia C and Negri E: European cancer mortality predictions for the year 2016 with focus on leukemias. Ann Oncol 27: 725-731, 2016

5. Brufsky AM: Long-term management of patients with hormone receptor-positive metastatic breast cancer: Concepts for sequential and combination endocrine-based therapies. Cancer Treat Rev 59: 22-32, 2017.

6. Majeed W, Aslam B, Javed I, Khaliq T, Muhammad F, Ali A and Raza A: Breast cancer: Major risk factors and recent developments in treatment. Asian Pac J Cancer Prev 15: 3353-3358, 2014

7. Jafari SH,SaadatpourZ, Salmaninejad A, Momeni F, Mokhtari M, Nahand JS, Rahmati M, Mirzaei H and Kianmehr M: Breast cancer diagnosis: Imaging techniques and biochemical markers. J Cell Physiol 233: 5200-5213, 2018.

8. Qu S, Liu Z, Yang X, Zhou J, Yu H, Zhang R and Li H: The emerging functions and roles of circular RNAs in cancer. Cancer Lett 414: 301-309, 2018

9. Han YN, Xia SQ, Zhang YY, Zheng JH and Li W: Circular RNAs: A novel type of biomarker and genetic tools in cancer. Oncotarget 8: 64551-64563, 2017.

10. Li Z, Chen Z, Hu G and Jiang Y: Roles of circular RNA in breast cancer: Present and future. Am J Transl Res 11: 3945-3954, 2019.

11. Zhou SY, Chen W, Yang SJ, Xu ZH, Hu JH, Zhang HD, Zhong SL and Tang JH: The emerging role of circular RNAs in breast cancer. Biosci Rep 39: BSR20190621, 2019.

12. Ng WL, MohdMohidin TB and Shukla K: Functional role of circular RNAs in cancer development and progression. RNA Biol 15: 995-1005, 2018

13. Lukiw WJ: Circular RNA (circRNA) in Alzheimer's disease (AD). Front Genet 4: 307, 2013.

14. Zhou J, Zhang WW, Peng F, Sun JY, He ZY and Wu SG: Downregulation of hsa_circ_0011946 suppresses the migration and invasion of the breast cancer cell line MCF-7 by targeting RFC3. Cancer Manag Res 10: 535-544, 2018.

15. Du WW, Yang W, Liu E, Yang Z, Dhaliwal P and Yang BB: Foxo3 circular RNA retards cell cycle progression via forming ternary complexes with p21 and CDK2. Nucleic Acids Res 44: 2846-2858, 2016.

16. Lü L, Sun J, Shi P, Kong W, Xu K, He B, Zhang S and Wang J: Identification of circular RNAs as a promising new class of diagnostic biomarkers for human breast cancer. Oncotarget 8: 44096-44107, 2017.

17. Li X, Ma F, Wu L, Zhang X, Tian J, Li J, Cao J, Ma Y, Zhang L and Wang L: Identification of Hsa_circ_0104824 as a potential biomarkers for breast cancer. Technol Cancer Res Treat 19 $1533033820960745,2020$.

18. Bian Q: Circular RNA PVT1 promotes the invasion and epithelial-mesenchymal transition of breast cancer cells through serving as a competing endogenous RNA for miR-204-5p. Onco Targets Ther 12: 11817-11826, 2019.

19. Yi Z, Li Y, Wu Y, Zeng B, Li H, Ren G and Wang X: Circular RNA 0001073 attenuates malignant biological behaviours in breast cancer cell and is delivered by nanoparticles to inhibit mice tumour growth. Onco Targets Ther 13: 6157-6169, 2020.

20. Tang YY, Zhao P, Zou TN, Duan JJ, Zhi R, Yang SY, Yang DC and Wang XL: Circular RNA hsa_circ 0001982 promotes breast cancer cell carcinogenesis through decreasing miR-143. DNA Cell Biol 36: 901-908, 2017.
21. Yin WB, Yan MG, Fang X, Guo JJ, Xiong W and Zhang RP. Circulating circular RNA hsa_circ_0001785 acts as a diagnostic biomarker for breast cancer detection. Clin Chim Acta 487: 363-368, 2018

22. Wu J, Jiang Z, Chen C, Hu Q, Fu Z, Chen J, Wang Z, Wang Q, Li A, Marks JR, et al: CircIRAK3 sponges miR-3607 to facilitate breast cancer metastasis. Cancer Lett 430: 179-192, 2018.

23. Hayes DF, Allred C, Anderson BO, et al: Breast. In: American Joint Committee on Cancer (AJCC) Cancer Staging Manual. 7th edition. Edge SB, Byrd DR and Compton CC (eds). Springer, New York, NY, pp347-376, 2009.

24. Livak KJ and Schmittgen TD: Analysis of relative gene expression data using real-time quantitative PCR and the 2(-Delta Delta C(T)) method. Methods 25: 402-408, 2001

25. Chekhun VF, Lukianova NY, Chekhun SV, Bezdieniezhnykh NO, Zadvorniy TV, Borikun TV, Polishchuk LZ and Klyusov OM: Association of $\mathrm{CD} 44^{+} \mathrm{CD} 24^{- \text {-low }}$ with markers of aggressiveness and plasticity of cell lines and tumors of patients with breast cancer. Exp Oncol 39: 203-211, 2017.

26. Tang T, Zhu Q, Li X, Zhu G, Deng S, Wang Y, Ni L, Chen X, Zhang Y, Xia T, et al: Protease Nexin I is a feedback regulator of EGF/PKC/MAPK/EGR1 signaling in breast cancer cells metastasis and stemness. Cell Death Dis 10: 649, 2019.

27. Croker AK, Goodale D, Chu J, Postenka C, Hedley BD, Hess DA and Allan AL: High aldehyde dehydrogenase and expression of cancer stem cell markers selects for breast cancer cells with enhanced malignant and metastatic ability. J Cell Mol Med 13: 2236-2252, 2009

28. Liu Z, Zhou Y, Liang G, Ling Y, Tan W, Tan L, Andrews R, Zhong W,Zhang X, Song E, et al: Circular RNA hsa_circ_001783 regulates breast cancer progression via sponging miR-200c-3p. Cell Death Dis 10: 55, 2019.

29. Tasharrofi B and Ghafouri-Fard S: Long non-coding RNAs as regulators of the mitogen-activated protein kinase (MAPK) pathway in cancer. Klin Onkol 31: 95-102, 2018.

30. Roversi FM, Olalla Saad ST and Machado-Neto JA: Serine peptidase inhibitor Kunitz type 2 (SPINT2) in cancer development and progression. Biomed Pharmacother 101: 278-286, 2018.

31. Nair AA, Niu N, Tang X, Thompson KJ, Wang L, Kocher JP, Subramanian S and Kalari KR: Circular RNAs and their associations with breast cancer subtypes. Oncotarget 7: 80967-80979, 2016.

32. Wang Y, Li J, Du C, Zhang L, Zhang Y, Zhang J and Wang L: Upregulated circular RNA circ-UBE2D2 predicts poor prognosis and promotes breast cancer progression by sponging miR-1236 and miR-1287. Transl Oncol 12: 1305-1313, 2019.

33. Martinez AM and Cavalli G: The role of polycomb group proteins in cell cycle regulation during development. Cell Cycle 5: 1189-1197, 2006.

34. Margueron R and Reinberg D: The polycomb complex PRC2 and its mark in life. Nature 469: 343-349, 2011.

35. Wang X, Hu B, Shen H, Zhou H, Xue X, Chen Y, Chen S, Han Y, Yuan B, Zhao H, et al: Clinical and prognostic relevance of EZH2 in breast cancer: A meta-analysis. Biomed Pharmacother 75 . 218-225, 2015.

36. Boostani F, Dolatkhah R, Fakhrjou A, Farassati F and Sanaat Z: Association of clinicopathologic characteristics and outcomes with EZH2 expression in patients with breast cancer in East Azerbaijan, Iran. Onco Targets Ther 11: 449-457, 2018

37. Shen L, Cui J, Liang S, Pang Y and Liu P: Update of research on the role of EZH2 in cancer progression. Onco Targets Ther 6: 321-324, 2013.

38. Pourakbar S, Pluard TJ, Accurso AD and Farassati F: Ezh2. A novel target in detection and therapy of breast cancer. Onco Targets Ther 10: 2685-2687, 2017.

39. Xue J, Liu Y, Luo F, Lu X, Xu H, Liu X, Lu L, Yang Q, Chen C, Fan W and Liu Q: Circ100284, via miR-217 regulation of EZH2, is involved in the arsenite-accelerated cell cycle of human keratinocytes in carcinogenesis. Biochim Biophys Acta Mol Basis Dis 1863: 753-763, 2017

40. Beca F, Kensler K, Glass B, Schnitt SJ, Tamimi RM and Beck AH: EZH2 protein expression in normal breast epithelium and risk of breast cancer: Results from the Nurses' health studies. Breast Cancer Res 19: 21, 2017.

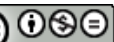

This work is licensed under a Creative Commons Attribution-NonCommercial-NoDerivatives 4.0 International (CC BY-NC-ND 4.0) License. 